\title{
PROLink - A single step circularization and purification procedure for the generation of an improved variant of human growth hormone
}

Nicolas Rasche ${ }^{\dagger, \ddagger}$, Jason Tonillo $\ddagger$, Marcel Rieker ${ }^{\dagger, \neq}$, Stefan Becker ${ }^{\ddagger}$, Brent Dorrll, Dmitry Ter-Ovanesyan§, Ulrich A.K. Betz ${ }^{\ddagger}$, Björn Hock ${ }^{\ddagger}$, Harald Kolmart, ${ }^{\dagger}$

†Institute for Organic Chemistry and Biochemistry, Technische Universität Darmstadt ¥Merck KGaA, Frankfurterstr. 250, 64293 Darmstadt, Germany \|present address: GlaxoSmithKline, Platform Technology \& Science, King of Prussia, PA 19406, United States

\$Department of Molecular and Cellular Biology, Harvard University, Cambridge MA 02138 and Wyss Institute for Biologically Inspired Engineering, Boston, MA 02115, United States

*E-mail: Kolmar@Biochemie-TUD 


\section{Supplementary Figures}

a

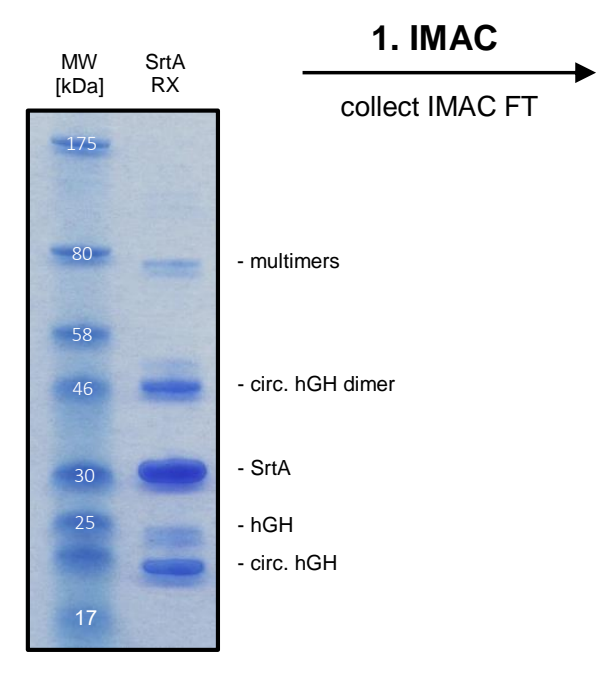

b

14

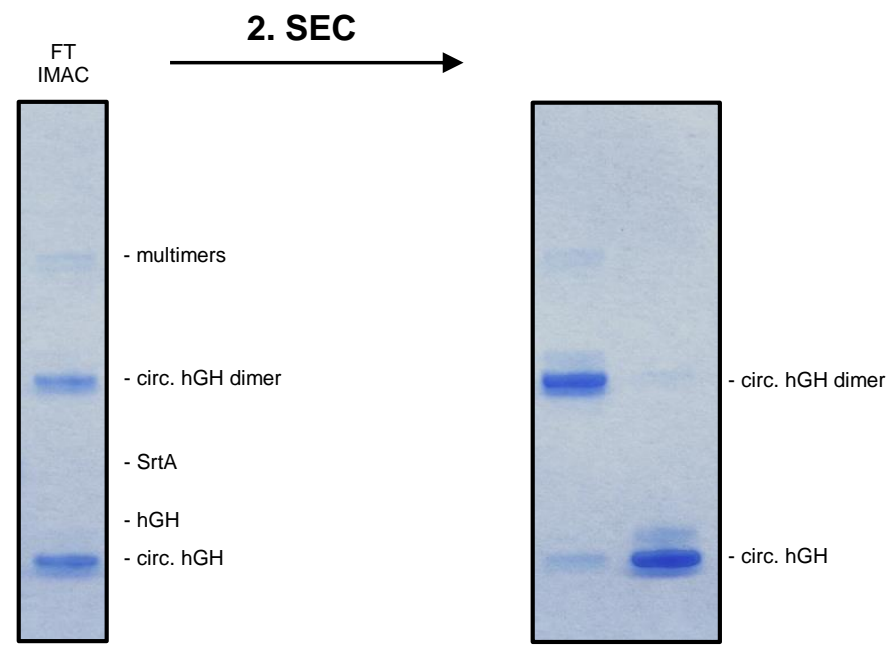

Figure S1. Purification of circular hGH. SDS-PAGE analysis of different purification stages. The sortase A reaction a) was passed through an IMAC column and the flow through b) was collected. Circular hGH monomers were separated from circular hGH dimers and multimers via SEC c).

a

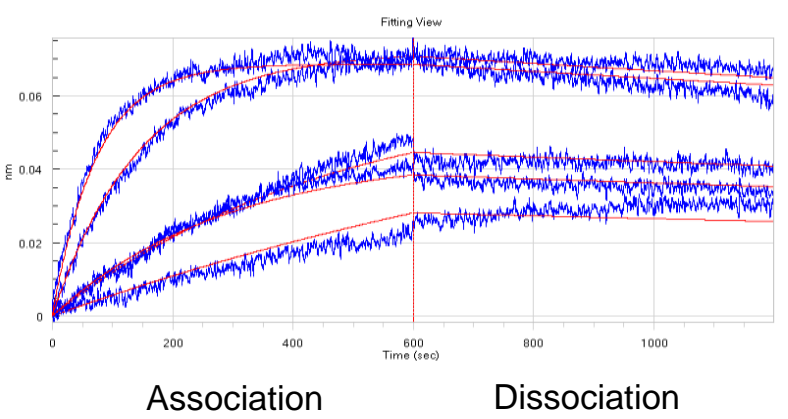

b

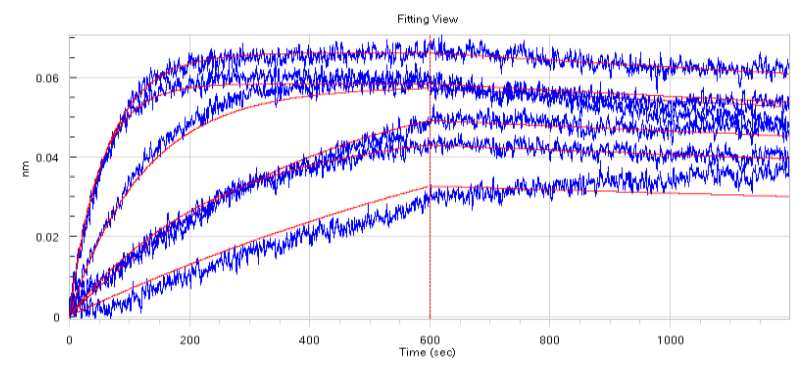

Association

Dissociation

Figure S2. Biolayer interferometry measurements of linear and circular hGH. A human growth hormone receptor $\mathrm{Fc}$ chimera was loaded onto $\mathrm{AHC}$ biosensors and association and dissociation of linear a) and circular hGH b) were analyzed. 


\section{a}

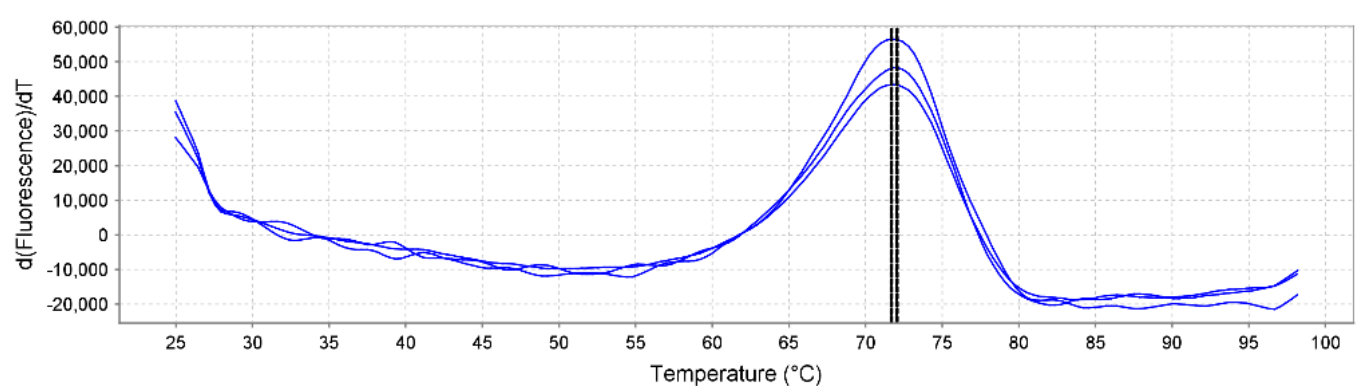

b

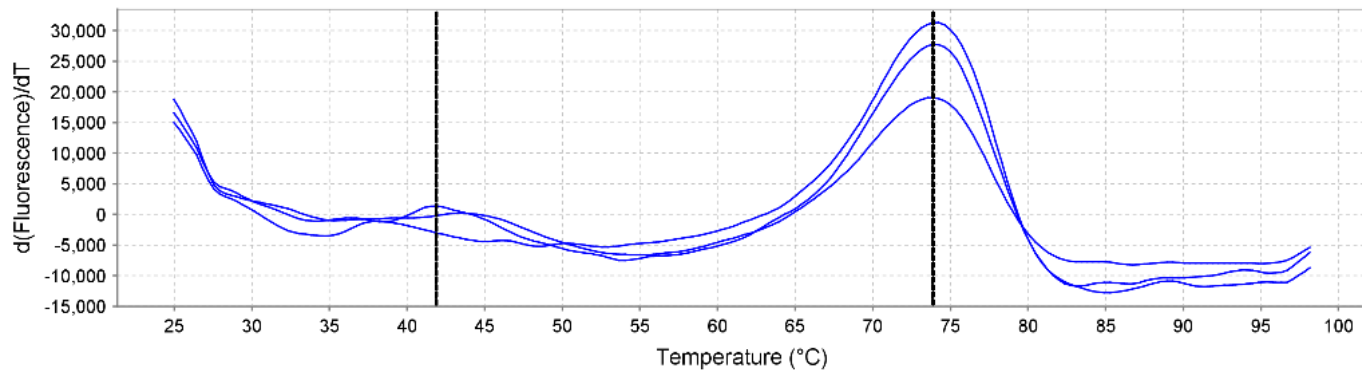

Figure S3. Differential scanning fluorimetry. First derivation of the melting curve obtained from DSF measurements of linear a) and circular hGH b). 
a

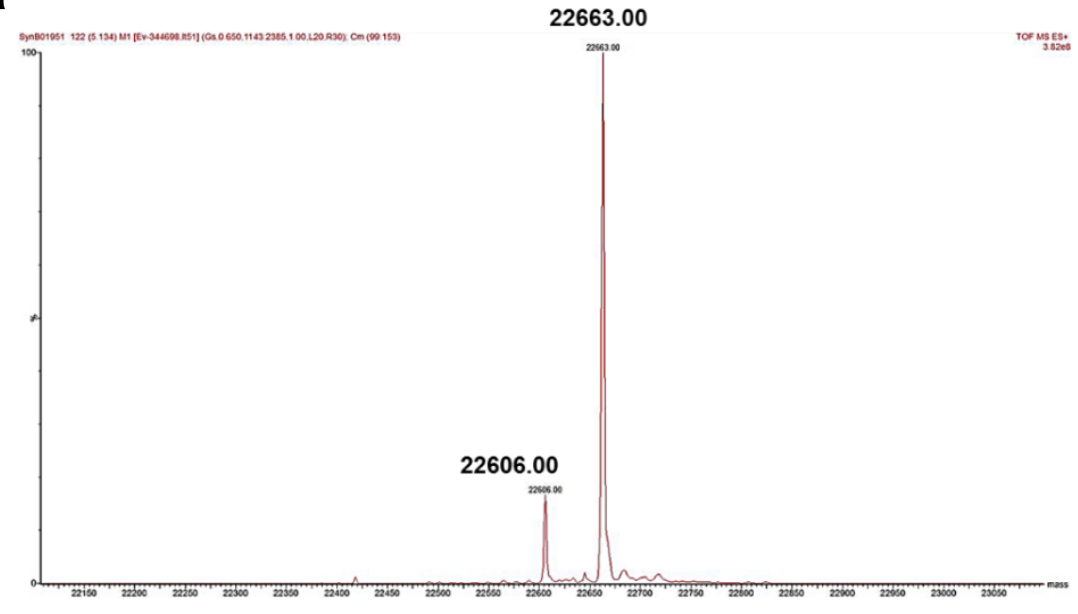

b SVEGSCGFLPETGGFPTIPLSR, $\mathrm{M}_{\mathrm{r}}=2251$

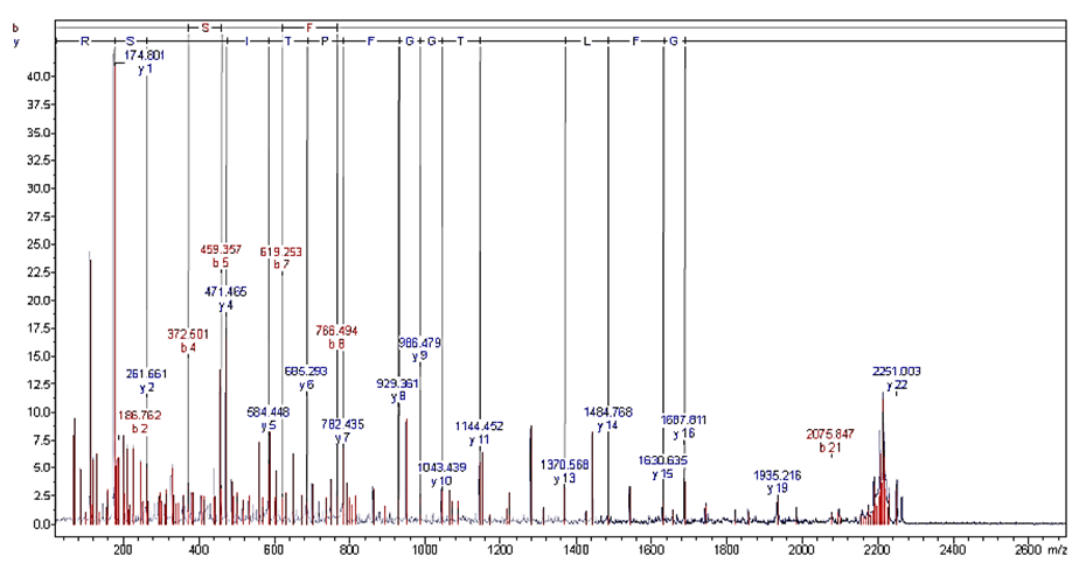

Figure S4. MS analysis of exoprotease treated hGH (a) MS characterization of purified circular monomeric hGH, intact mass 22663 (calculated 22665). (b) MS/MS identification of a tryptic peptide comprising the hGH $C$-terminus with the SitA motif fused to the $N$-terminal glycine. 